\title{
Sharp inequalities involving the symmetric mean
}

Tiberiu Trif 


\title{
SHARP INEQUALITIES INVOLVING THE SYMMETRIC MEAN
}

\author{
TiBERIU TRIF \\ Babeş - Bolyai University, Department of Mathematics \\ 1, M. Kogălniceanu St., 3400 Cluj-Napoca, Romania \\ ttrif@math.ubbcluj.ro
}

[Received: September 9, 2001]

\begin{abstract}
In this paper we prove several sharp inequalities involving the symmetric mean of two arguments. They refine already known inequalities and relate to Hölder means as well as to some special Gini means.
\end{abstract}

Mathematical Subject Classification: 26D99

Keywords: means, symmetric mean, inequalities

\section{Introduction and notation}

Given the positive real numbers $x$ and $y$, let $A(x, y):=\frac{x+y}{2}, G(x, y):=\sqrt{x y}$,

$$
L(x, y):=\left\{\begin{array}{cl}
\frac{x-y}{\ln x-\ln y} & \text { if } x \neq y \\
x & \text { if } x=y
\end{array}\right.
$$

and

$$
H_{p}(x, y):=\left\{\begin{array}{cc}
\left(\frac{x^{p}+y^{p}}{2}\right)^{1 / p} & \text { if } p \neq 0 \\
\sqrt{x y} & \text { if } p=0
\end{array}\right.
$$

denote their arithmetic, geometric, logarithmic, and Hölder means, respectively. It is well known that if $x \neq y$, then $H_{p}$ is strictly increasing in $p$. The symmetric mean of $x$ and $y$ is defined by (see [4, pp. 44-45])

$$
S_{\alpha}(x, y):=\frac{x^{\alpha} y^{1-\alpha}+x^{1-\alpha} y^{\alpha}}{2} \quad \alpha \in \mathbf{R} .
$$

Since $S_{\alpha}(x, y)=S_{1-\alpha}(x, y)$, it suffices to consider $\alpha \geq 1 / 2$. Following A. O. Pittenger [8], we write the symmetric mean into the form

$$
S_{\delta}(x, y):=\frac{x^{\frac{1+\sqrt{\delta}}{2}} y^{\frac{1-\sqrt{\delta}}{2}}+x^{\frac{1-\sqrt{\delta}}{2}} y^{\frac{1+\sqrt{\delta}}{2}}}{2} \quad \delta \geq 0 .
$$

It is easily seen that if $x \neq y$, then $S_{\delta}$ is strictly increasing in $\delta$, so

$$
G(x, y)=S_{0}(x, y)<S_{\delta}(x, y)<S_{1}(x, y)=A(x, y)
$$

for all $\delta \in] 0,1[$. 
A. O. Pittenger [8, Theorem 1 and Theorem 2] proved that for all positive real numbers $x \neq y$, the symmetric mean satisfies also the following inequalities:

$$
\begin{array}{ll}
S_{1 / 3}(x, y)<L(x, y), & \\
S_{\delta}(x, y)<H_{\delta}(x, y) & \text { if } 0<\delta<1, \\
S_{\delta}(x, y)>H_{\delta}(x, y) & \text { if } \delta>1 .
\end{array}
$$

At this point we present a very short proof of the intriguing inequality (1.2), which is sharp in the sense that $1 / 3$ cannot be replaced by any greater constant. In order to prove (1.2), let us apply the Gauss quadrature formula with two knots (see [1, pp. $343-344]$, [2, p. 36])

$$
\int_{0}^{1} f(t) d t=\frac{1}{2} f\left(\frac{1}{2}+\frac{1}{2 \sqrt{3}}\right)+\frac{1}{2} f\left(\frac{1}{2}-\frac{1}{2 \sqrt{3}}\right)+\frac{1}{4320} f^{(4)}(\xi) \quad 0<\xi<1,
$$

to the function $f(t)=x^{t} y^{1-t}$. Since $\int_{0}^{1} f(t) d t=L(x, y)$ (this integral representation of $L(x, y)$ was pointed out for the first time by E. Neuman [6]), we get

$$
L(x, y)=S_{1 / 3}(x, y)+\frac{1}{4320} x^{\xi} y^{1-\xi}(\ln x-\ln y)^{4} \quad 0<\xi<1 .
$$

Therefore

$$
\frac{\min (x, y)}{4320}(\ln x-\ln y)^{4}<L(x, y)-S_{1 / 3}(x, y)<\frac{\max (x, y)}{4320}(\ln x-\ln y)^{4} .
$$

This inequality yields (1.2) and estimates the sharpness of (1.2).

It is the main purpose of the present paper to point out other sharp inequalities involving the symmetric mean. In Section 2 we are concerned with sharp "interpolating inequalities" related to (1.1) and (1.2). In Section 3 we establish two sharp inequalities involving the symmetric and special Gini means.

\section{Sharp interpolating inequalities related to (1.1) and (1.2)}

In the next theorem we prove a sharp interpolating inequality related to (1.1):

TheORem 2.1. If $0<\delta<1$, then for all positive real numbers $x \neq y$ it holds that

$$
S_{\delta}(x, y)<\delta A(x, y)+(1-\delta) G(x, y) .
$$

Moreover, (2.1) is the best possible inequality of the type

$$
S_{\delta}(x, y)<\lambda A(x, y)+(1-\lambda) G(x, y) \quad 0<\lambda<1,
$$

in the sense that if $0<\lambda<\delta$, then (2.2) cannot be true for all positive real numbers $x \neq y$.

Proof. Letting $x / y=e^{2 t}(t \in \mathbf{R})$ and multiplying both sides by $e^{-t}$, we see that (2.2) is equivalent to $f_{\lambda}(t)<0$ for all $t \neq 0$, where $f_{\lambda}: \mathbf{R} \rightarrow \mathbf{R}$ is the function defined by $f_{\lambda}(t):=\cosh (\sqrt{\delta} t)-\lambda \cosh t-1+\lambda$. By using the power series expansion

$$
\cosh z=1+\sum_{n=1}^{\infty} \frac{1}{(2 n) !} z^{2 n}
$$


we get

$$
f_{\lambda}(t)=\sum_{n=1}^{\infty} \frac{a_{n}(\lambda)}{(2 n) !} t^{2 n}, \quad \text { where } \quad a_{n}(\lambda):=\delta^{n}-\lambda .
$$

If $0<\lambda<\delta$, then $a_{1}(\lambda)=\delta-\lambda>0$, hence $f_{\lambda}(t)>0$ for $|t|$ sufficiently small. Therefore, (2.2) cannot be true for all positive real numbers $x \neq y$.

On the other hand, $a_{1}(\delta)=0$ and $a_{n}(\delta)<0$ for all $n \geq 2$. Consequently, $f_{\delta}(t)<0$ for all $t \neq 0$. This proves the validity of (2.1).

Remark. Obviously, (2.1) is a refinement of the second inequality in (1.1). It would be also interesting to compare (2.1) with (1.3). Thus, for $0<\delta<1 / 2$ the inequality (2.1) is weaker than (1.3), for $\delta=1 / 2$ it coincides with (1.3), while for $1 / 2<\delta<1$ it is stronger than (1.3). This assertion is proved by the following theorem.

Theorem 2.2. For all positive real numbers $x \neq y$ it holds that

$$
\delta A(x, y)+(1-\delta) G(x, y)>H_{\delta}(x, y) \quad \text { if } \quad 0<\delta<1 / 2
$$

and

$$
\delta A(x, y)+(1-\delta) G(x, y)<H_{\delta}(x, y) \quad \text { if } \quad 1 / 2<\delta<1 .
$$

Proof. Due to the symmetry, we may assume in both (2.4) and (2.5) that $x>y$. Letting $x / y=e^{2 t}(t>0)$ and multiplying both sides by $e^{-t}$, we see that (2.4) is equivalent to $f_{\delta}(t)>1$ for all $t>0$, where $f_{\delta}: \mathbf{R} \rightarrow \mathbf{R}$ is the function defined by

$$
f_{\delta}(t):=\frac{\delta \cosh t+1-\delta}{(\cosh (\delta t))^{1 / \delta}} \text {. }
$$

We have

$$
f_{\delta}^{\prime}(t)=\frac{g(t)}{(\cosh (\delta t))^{1+1 / \delta}}, \quad \text { where } \quad g(t)=\delta \sinh ((1-\delta) t)-(1-\delta) \sinh (\delta t) .
$$

By using the power series expansion

$$
\sinh z=\sum_{n=0}^{\infty} \frac{1}{(2 n+1) !} z^{2 n+1}
$$

we get

$$
g(t)=\sum_{n=1}^{\infty} \frac{\delta(1-\delta)\left[(1-\delta)^{2 n}-\delta^{2 n}\right]}{(2 n+1) !} t^{2 n+1} .
$$

Since $0<\delta<1 / 2$, it follows that $g(t)>0$ for all $t>0$, hence $f_{\delta}$ is strictly increasing on ] $0, \infty\left[\right.$. Therefore $f_{\delta}(t)>1$ for all $t>0$, because $f_{\delta}(0)=1$. This completes the proof of (2.4). The proof of (2.5) is analogous.

From (1.1) and (1.2) it follows that $G(x, y)<S_{\delta}(x, y)<L(x, y)$ for all $0<\delta \leq 1 / 3$. Related to this, the following sharp interpolating inequality holds. 
TheOREM 2.3. If $0<\delta \leq 1 / 3$, then for all positive real numbers $x \neq y$ it holds that

$$
S_{\delta}(x, y)<3 \delta L(x, y)+(1-3 \delta) G(x, y) .
$$

Moreover, (2.7) is the best possible inequality of the type

$$
S_{\delta}(x, y)<\lambda L(x, y)+(1-\lambda) G(x, y) \quad 0<\lambda \leq 1,
$$

in the sense that if $0<\lambda<3 \delta$, then (2.8) cannot be true for all positive real numbers $x \neq y$.

Proof. Due to the symmetry, we may assume in both (2.7) and (2.8) that $x>y$. Letting $x / y=e^{2 t}(t>0)$ and multiplying both sides by $t e^{-t}$, we see that (2.8) is equivalent to $f_{\lambda}(t)<0$ for all $t>0$, where $\left.f_{\lambda}:\right] 0, \infty[\rightarrow \mathbf{R}$ is the function defined by $f_{\lambda}(t):=t \cosh (\sqrt{\delta} t)-\lambda \sinh t-(1-\lambda) t$. From (2.3) and (2.6) we get

$$
f_{\lambda}(t)=\sum_{n=1}^{\infty} \frac{a_{n}(\lambda)}{(2 n+1) !} t^{2 n+1}, \quad \text { where } \quad a_{n}(\lambda):=(2 n+1) \delta^{n}-\lambda .
$$

If $0<\lambda<3 \delta$, then $a_{1}(\lambda)=3 \delta-\lambda>0$, hence $f_{\lambda}(t)>0$ for $t>0$ sufficiently small. Therefore, (2.8) cannot be true for all positive real numbers $x \neq y$.

On the other hand, $a_{1}(3 \delta)=0$ and $a_{n}(3 \delta)=\delta\left[(2 n+1) \delta^{n-1}-3\right]<0$ for all $n \geq 2$, because

$$
(2 n+1) \delta^{n-1}-3 \leq \frac{2 n+1}{3^{n-1}}-3<0 \quad \text { for all } n \geq 2 .
$$

The inequality $3^{n}>2 n+1(n \geq 2)$ can be easily proved by induction. Consequently, $f_{3 \delta}(t)<0$ for all $t>0$. This proves the validity of $(2.7)$.

Remark. The inequality (2.7) is a refinement of (2.1), because

$$
3 \delta L(x, y)+(1-3 \delta) G(x, y)<\delta A(x, y)+(1-\delta) G(x, y) .
$$

Indeed, this inequality is equivalent to

$$
L(x, y)<\frac{1}{3} A(x, y)+\frac{2}{3} G(x, y),
$$

a well known inequality due to E. B. Leach and M. C. Sholander [5].

From (1.1) and (1.3) it follows that $G(x, y)<S_{\delta}(x, y)<H_{\delta}(x, y)$ for all $0<\delta<1$. It is not difficult to see that we cannot have a sharp inequality of the type

$$
S_{\delta}(x, y)<\lambda H_{\delta}(x, y)+(1-\lambda) G(x, y) \quad 0<\lambda<1 .
$$

\section{Sharp inequalities involving the symmetric and special Gini means}

Given the positive real numbers $x$ and $y$, the Lehmer mean $L_{r}(x, y)$ of $x$ and $y$ is defined for each $r \in \mathbf{R}$ by (see [9])

$$
L_{r}(x, y):=\frac{x^{r+1}+y^{r+1}}{x^{r}+y^{r}} .
$$


In this section we deal also with the mean

$$
M(x, y):=\exp \left(\frac{x \ln x+y \ln y}{x+y}\right)=x^{x /(x+y)} y^{y /(x+y)} .
$$

It should be noted that both the Lehmer means and the mean $M$ are special cases of Gini means (see [3], [7]).

In the next theorem a sharp inequality involving the symmetric and Lehmer means is established.

THEOREM 3.1. If $r>0$, then for all positive real numbers $x \neq y$ it holds that

$$
S_{2 r+1}(x, y)>L_{r}(x, y) .
$$

Moreover, (3.1) is the best possible inequality of the type

$$
S_{\delta}(x, y)>L_{r}(x, y)
$$

in the sense that if $0<\delta<2 r+1$, then (3.2) cannot be true for all positive real numbers $x \neq y$.

Proof. Letting $x / y=e^{2 t}(t \in \mathbf{R})$, after a little bit of algebra we see that

$$
\begin{aligned}
\frac{S_{\delta}(x, y)}{L_{r}(x, y)}-1 & =\frac{\left[\left(\frac{x}{y}\right)^{\frac{1+\sqrt{\delta}}{2}}+\left(\frac{x}{y}\right)^{\frac{1-\sqrt{\delta}}{2}}\right]\left[\left(\frac{x}{y}\right)^{r}+1\right]}{2\left[\left(\frac{x}{y}\right)^{r+1}+1\right]} \\
& =\frac{\cosh (\sqrt{\delta} t) \cosh (r t)}{\cosh ((r+1) t)}-1 \\
& =\frac{f_{\delta}(t)}{2 \cosh ((r+1) t)}
\end{aligned}
$$

where $f_{\delta}: \mathbf{R} \rightarrow \mathbf{R}$ is the function defined by

$$
f_{\delta}(t):=\cosh ((r+\sqrt{\delta}) t)+\cosh ((r-\sqrt{\delta}) t)-2 \cosh ((r+1) t) .
$$

By using the power series expansion (2.3) we get

$f_{\delta}(t)=\sum_{n=1}^{\infty} \frac{a_{n}(\delta)}{(2 n) !} t^{2 n}, \quad$ where $\quad a_{n}(\delta):=(r+\sqrt{\delta})^{2 n}+(r-\sqrt{\delta})^{2 n}-2(r+1)^{2 n}$.

Suppose first that $0<\delta<2 r+1$. Since $a_{1}(\delta)=2(\delta-2 r-1)$, it follows that $f_{\delta}(t)<0$ for $|t|$ sufficiently small. Consequently, $S_{\delta}(x, y)<L_{r}(x, y)$ for $x \neq y$ sufficiently close, hence (3.2) cannot be true for all positive real numbers $x \neq y$.

Next we show that (3.1) holds true. Set, for brevity,

$$
a_{n}=a_{n}(2 r+1)=(r+\sqrt{2 r+1})^{2 n}+(r-\sqrt{2 r+1})^{2 n}-2(r+1)^{2 n}
$$

for each positive integer $n$. Then $a_{1}=0$ and, in order to establish the validity of (3.1), it suffices to prove that $a_{n}>0$ for every $n \geq 2$. 
Set $x_{n}:=(r+\sqrt{2 r+1})^{2 n}+(r-\sqrt{2 r+1})^{2 n}$ for each positive integer $n$. Then the sequence $\left(x_{n}\right)$ satisfies

$$
x_{n+1}=2(r+1)^{2} x_{n}-\left(r^{2}-2 r-1\right)^{2} x_{n-1} \quad \text { for every } n \geq 2 .
$$

We claim that

$$
(r+1)^{2} x_{n}>\left(r^{2}-2 r-1\right)^{2} x_{n-1}
$$

for all $n \geq 2$. Since $x_{1}=2(r+1)^{2}$ and $x_{2}=(r+\sqrt{2 r+1})^{4}+(r-\sqrt{2 r+1})^{4}$, for $n=2$ the inequality (3.4) is equivalent to

$(r+\sqrt{2 r+1})^{4}+(r-\sqrt{2 r+1})^{4}>2\left(r^{2}-2 r-1\right)^{2}=2(r+\sqrt{2 r+1})^{2}(r-\sqrt{2 r+1})^{2}$, which is true because $u^{2}+v^{2}>2 u v$ for $u \neq v$. Assuming that (3.4) holds for $n \geq 2$, we prove that it holds also for $n+1$. By virtue of (3.3) we have

$$
\begin{aligned}
(r+1)^{2} x_{n+1}-\left(r^{2}-2 r-1\right)^{2} x_{n} \\
\quad=\left[2(r+1)^{4}-\left(r^{2}-2 r-1\right)^{2}\right] x_{n}-(r+1)^{2}\left(r^{2}-2 r-1\right)^{2} x_{n-1} \\
\quad=\left(r^{4}+12 r^{3}+10 r^{2}+4 r+1\right) x_{n}-(r+1)^{2}\left(r^{2}-2 r-1\right)^{2} x_{n-1} \\
\quad>(r+1)^{4} x_{n}-(r+1)^{2}\left(r^{2}-2 r-1\right)^{2} x_{n-1} \\
\quad>0 .
\end{aligned}
$$

Consequently, (3.4) holds true as claimed.

Next, we prove by induction that

$$
x_{n}>2(r+1)^{2 n}
$$

for all $n \geq 2$. Indeed, $x_{2}=2(r+1)^{4}+16 r^{3}+8 r^{2}>2(r+1)^{4}$. Assuming that (3.5) holds for $n \geq 2$, we show that it holds also for $n+1$. By virtue of (3.3) and (3.4) we have

$$
x_{n+1}>(r+1)^{2} x_{n}>2(r+1)^{2 n+2} .
$$

From (3.5) it follows that $a_{n}>0$ for every $n \geq 2$, completing the proof of the theorem.

REMARKS. 1. The inequality (3.1) is a refinement of $S_{2 r+1}(x, y)>H_{2 r+1}(x, y)$ (see (1.4)), by virtue of the inequality $L_{r}(x, y)>H_{2 r+1}(x, y)$ (see Z. Páles [7, Theorem 3], K. B. Stolarski [9, Theorem 1]).

2. For $-1 / 2<r<0$ the means $S_{2 r+1}$ and $L_{r}$ are not comparable. Indeed, with the notations in the proof of Theorem 3.1, we have $a_{1}=0$ and $a_{2}=8 r^{2}(2 r+1)<0$, hence $f_{2 r+1}(t)<0$ for $|t|$ sufficiently small. Consequently, $S_{2 r+1}(x, y)<L_{r}(x, y)$ for $x \neq y$ sufficiently close. On the other hand, since $\frac{1+\sqrt{2 r+1}}{2}>r+1$, it follows that

$$
\frac{S_{2 r+1}(x, y)}{L_{r}(x, y)}=\frac{\left[\left(\frac{x}{y}\right)^{\frac{1+\sqrt{2 r+1}}{2}}+\left(\frac{x}{y}\right)^{\frac{1-\sqrt{2 r+1}}{2}}\right]\left[\left(\frac{x}{y}\right)^{r}+1\right]}{2\left[\left(\frac{x}{y}\right)^{r+1}+1\right]}>1
$$

for $x / y$ sufficiently large.

Now we prove a sharp inequality involving the means $S_{\delta}$ and $M$. 
TheOREM 3.2. For all positive real numbers $x \neq y$ it holds that

$$
S_{2}(x, y)>M(x, y) .
$$

Moreover, (3.6) is the best possible inequality of the type

$$
S_{\delta}(x, y)>M(x, y),
$$

in the sense that if $0 \leq \delta<2$, then (3.7) cannot be true for all positive real numbers $x \neq y$.

Proof. Letting $x / y=e^{2 t}(t \in \mathbf{R})$ and multiplying both sides by $e^{-t}$, we see that (3.7) is equivalent to $f_{\delta}(t)>0$ for all $t \neq 0$, where $f_{\delta}: \mathbf{R} \rightarrow \mathbf{R}$ is the function defined by $f_{\delta}(t):=\ln (\cosh (\sqrt{\delta} t))-t \tanh t$. We have

$$
\tanh z=\sum_{n=1}^{\infty} \frac{2^{2 n}\left(2^{2 n}-1\right)}{(2 n) !} B_{2 n} z^{2 n-1} \quad|z|<\frac{\pi}{4}
$$

and

$$
\ln (\cosh z)=\sum_{n=1}^{\infty} \frac{2^{2 n}\left(2^{2 n}-1\right)}{2 n(2 n) !} B_{2 n} z^{2 n} \quad|z|<\frac{\pi}{4},
$$

where $B_{k}$ is the $k$ th Bernoulli number. Consequently, for $|t|$ sufficiently small, the inequality $f_{\delta}(t)>0$ is equivalent to

$$
\sum_{n=1}^{\infty} \frac{2^{2 n}\left(2^{2 n}-1\right)}{2 n(2 n) !} B_{2 n} \delta^{n} t^{2 n}>\sum_{n=1}^{\infty} \frac{2^{2 n}\left(2^{2 n}-1\right)}{(2 n) !} B_{2 n} t^{2 n} .
$$

If $0 \leq \delta<2$, then the last inequality cannot be true for all $t \neq 0$ with $|t|$ sufficiently small, because the coefficient of $t^{2}$ in the right side is greater than the coefficient of $t^{2}$ in the left side. Therefore, (3.7) cannot be true for all positive real numbers $x \neq y$.

Next we show that (3.6) holds true. We have just seen that (3.6) is equivalent to

$$
f(t):=f_{2}(t)=\ln (\cosh (\sqrt{2} t))-t \tanh t>0 \quad \text { for all } t \neq 0 .
$$

We have

$$
f^{\prime}(t)=\sqrt{2} \frac{\sinh (\sqrt{2} t)}{\cosh (\sqrt{2} t)}-\frac{\sinh t}{\cosh t}-\frac{t}{\cosh ^{2} t}=\frac{g(t)}{\cosh (\sqrt{2} t) \cosh ^{2} t},
$$

where $g(t)=\sqrt{2} \sinh (\sqrt{2} t) \cosh ^{2} t-\sinh t \cosh t \cosh (\sqrt{2} t)-t \cosh (\sqrt{2} t)$. By means of certain usual hyperbolic identities we obtain

$$
g(t)=\frac{\sinh (\sqrt{2} t)}{\sqrt{2}}-t \cosh (\sqrt{2} t)+\frac{2-\sqrt{2}}{4 \sqrt{2}} \sinh ((2+\sqrt{2}) t)-\frac{2+\sqrt{2}}{4 \sqrt{2}} \sinh ((2-\sqrt{2}) t) .
$$

Taking into account the power series expansions (2.3) and (2.6) we get

$$
g(t)=\sum_{n=0}^{\infty} \frac{a_{n}}{(2 n+1) !} t^{2 n+1}, \quad \text { where } \quad a_{n}:=\frac{(2+\sqrt{2})^{2 n}-(2-\sqrt{2})^{2 n}}{2 \sqrt{2}}-n 2^{n+1} .
$$


Note that $a_{1}=0$ and that

$$
\begin{aligned}
a_{n} & >\frac{(2+\sqrt{2})^{2 n}-1}{2 \sqrt{2}}-n 2^{n+1}>\frac{2^{2 n}+2 n \sqrt{2} 2^{2 n-1}-1}{2 \sqrt{2}}-n 2^{n+1} \\
& =\frac{2^{2 n}-1+2 n \sqrt{2}\left(2^{2 n-1}-2^{n+1}\right)}{2 \sqrt{2}}>0
\end{aligned}
$$

for all $n \geq 2$. Therefore, $g(t)<0$ for $t<0$ and $g(t)>0$ for $t>0$, hence $f^{\prime}(t)<0$ for $t<0$ and $f^{\prime}(t)>0$ for $t>0$. Consequently, $f$ is strictly decreasing on $]-\infty, 0[$ and strictly increasing on $] 0, \infty[$. Since $f(0)=0$, we can conclude that (3.8) holds true.

Remarks. 1. The inequality (3.6) is a refinement of $S_{2}(x, y)>L_{1 / 2}(x, y)$ (see (3.1)), by virtue of the inequality $M(x, y)>L_{1 / 2}(x, y)$ (see Z. Páles [7, Theorem 3]).

2. From (3.8) we deduce that

$$
\frac{x}{\sqrt{2}} \tanh \frac{x}{\sqrt{2}} \leq \ln (\cosh x) \quad \text { for all } x \in \mathbf{R} .
$$

This inequality is complementary to the inequality

$$
\ln (\cosh x) \leq x \tanh \frac{x}{2} \quad \text { for all } x \in \mathbf{R},
$$

established by K. B. Stolarsky [9, Theorem 3].

\section{REFERENCES}

[1] Davis, P. J.: Interpolation and Approximation, Blaisdell, New York, 1963.

[2] Davis, P. J. and Rabinowitz, P.: Numerical Integration, Blaisdell, MassachusettsToronto-London, 1967.

[3] Gini C.: Di una formula comprensiva delle medie, Metron, 13, (1938), 3-22.

[4] G. H. Hardy, G. H., Littlewood, J. E. and Pólya, G.: Inequalities, Cambridge Univ. Press, Cambridge, 1934.

[5] Leach, E. B. and Sholander, M. C.: Extended mean values II, J. Math. Anal. Appl., 92, (1983), 207-223.

[6] Neuman, E.: The weighted logarithmic mean, J. Math. Anal. Appl., 188, (1994), 885900.

[7] PÁles, Z.: Inequalities for sums of powers, J. Math. Anal. Appl., 131, (1988), 265-270.

[8] Pittenger, A. O.: The symmetric, logarithmic and power means, Univ. Beograd. Publ. Elektrotehn. Fak., Ser. Mat. Fiz., No. 681, (1980), 19-23.

[9] Stolarsky, K. B.: Hölder means, Lehmer means, and $x^{-1} \log \cosh x$, J. Math. Anal. Appl., 202, (1996), 810-818. 